\title{
LA-UR-20-23485
}

Approved for public release; distribution is unlimited.

Title: $\quad$ LANL \& UCLA Publication Collaboration, 2009-2018.

Author(s): $\quad$ Guthormsen, Amy Maria Johnson, Nicole M.

McKinley, Melanie Peters

Robinson, Melissa A.

Dushdurova, Valida

Intended for: Breefing

Issued:

2020-05-10 
Disclaimer:

Los Alamos National Laboratory, an affirmative action/equal opportunity employer, is operated by Triad National Security, LLC for the National Nuclear Security Administration of U.S. Department of Energy under contract 89233218CNA000001. By approving this article, the publisher recognizes that the U.S. Government retains nonexclusive, royalty-free license to publish or reproduce the published form of this contribution, or to allow others to do so, for U.S. Government purposes. Los Alamos National Laboratory requests that the publisher identify this article as work performed under the auspices of the U.S. Department of Energy. Los Alamos National Laboratory strongly supports academic freedom and a researcher's right to publish; as an institution, however, the Laboratory does not endorse the viewpoint of a publication or guarantee its technical correctness. 


\section{LANL \& UCLA Publication Collaboration, 2009-2018}

Amy Guthormsen Nicole Johnson Melanie McKinley Melissa Robinson Valida Dushdurova SRO-CP

- Los Alamos May 7, 2020 NATIONAL LABORATORY 


\section{Co-authored Publications, 2009 to 2018}

Collaboration with the University of California at Los Angeles

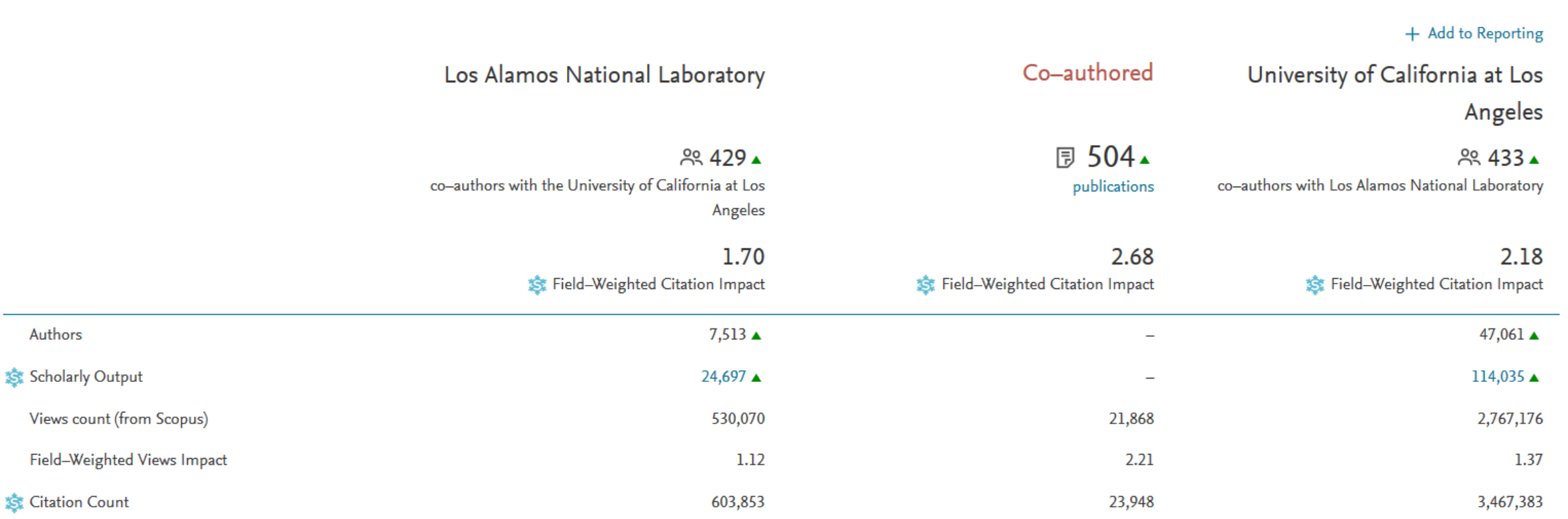




\section{Co-authored Publications by Subject Area}

Physics and Astronomy

Earth and Planetary Science... Agricultural and Biological...

Environmental Science

Materials Science

Engineering

Mathematics

Chemistry

Computer Science

Multidisciplinary

Biochemistry, Genetics and ...

Chemical Engineering Energy

Immunology and Microbiology

Medicine

Neuroscience

Social Sciences

Arts and Humanities

Psychology

Decision Sciences

Pharmacology, Toxicology an...

Business, Management and Ac..

Health Professions

$$
\text { Nursing }
$$

Economics, Econometrics and...

Veterinary

Dentistry
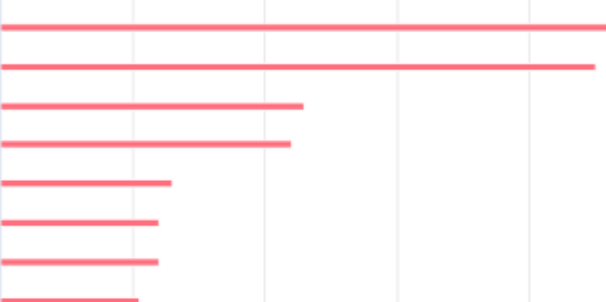


\section{All Topic Clusters for Co-authored Publications}

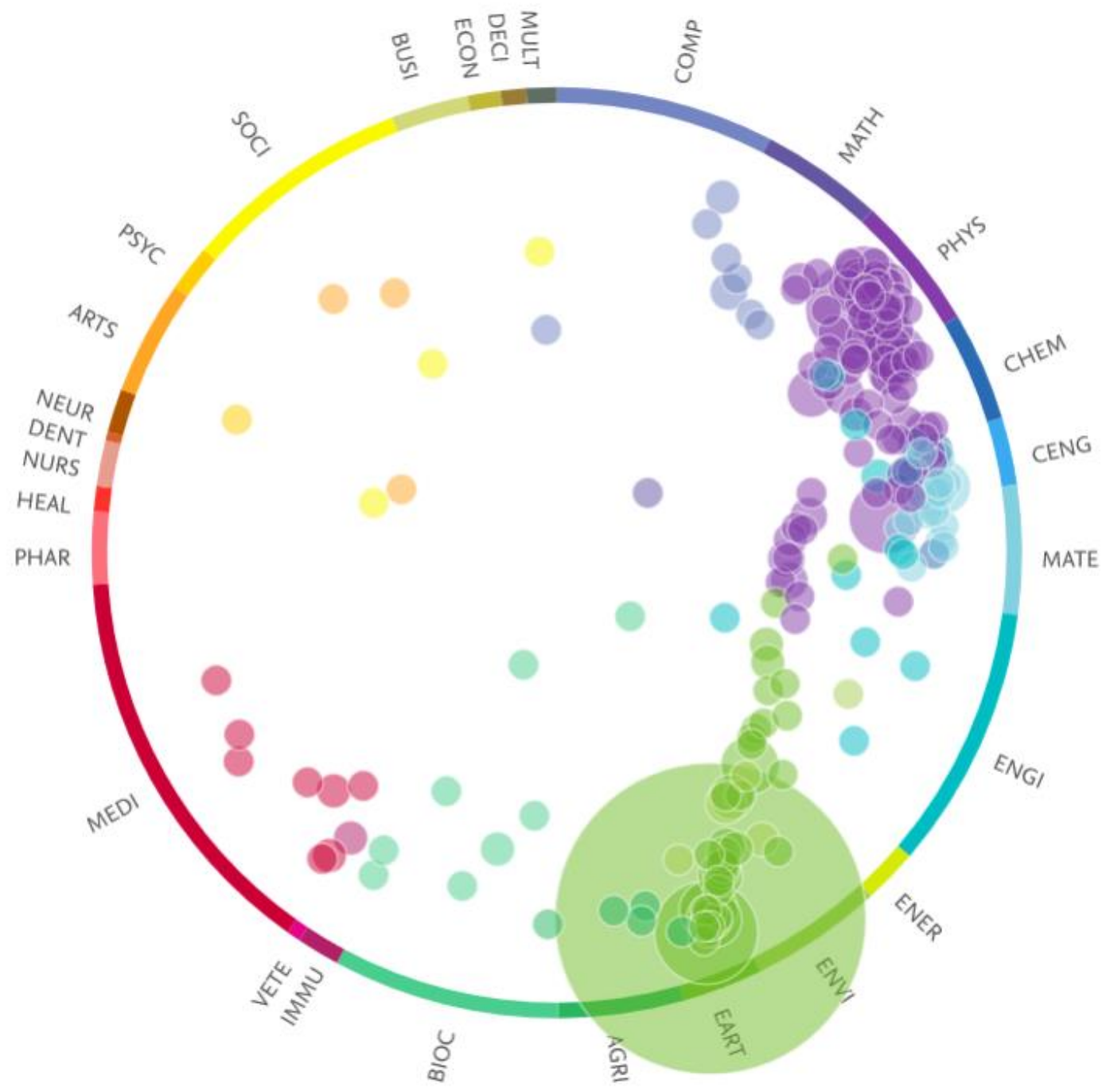

^ Subject area abbreviations

$\begin{array}{ll}\text { COMP } & \text { Computer Science } \\ \text { MATH } & \text { Mathematics } \\ \text { PHYS } & \text { Physics and Astronomy } \\ \text { CHEM } & \text { Chemistry } \\ \text { CENG } & \text { Chemical Engineering } \\ \text { MATE } & \text { Materials Science } \\ \text { ENGI } & \text { Engineering } \\ \text { ENER } & \text { Energy } \\ \text { ENVI } & \text { Environmental Science } \\ \text { EART } & \text { Earth and Planetary Sciences } \\ \text { AGRI } & \text { Agricultural and Biological Sciences } \\ \text { BIOC } & \text { Biochemistry, Genetics and Molecular Biology } \\ \text { IMMU } & \text { Immunology and Microbiology } \\ \text { VETE } & \text { Veterinary } \\ \text { MEDI } & \text { Medicine } \\ \text { PHAR } & \text { Pharmacology, Toxicology and Pharmaceutics } \\ \text { HEAL } & \text { Health Professions } \\ \text { NURS } & \text { Nursing } \\ \text { DENT } & \text { Dentistry } \\ \text { NEUR } & \text { Neuroscience } \\ \text { ARTS } & \text { Arts and Humanities } \\ \text { PSYC } & \text { Psychology } \\ \text { SOCI } & \text { Social Sciences } \\ \text { BUSI } & \text { Business, Management and Accounting } \\ \text { ECON } & \text { Economics, Econometrics and Finance } \\ \text { DECI } & \text { Decision Sciences } \\ \text { MULT } & \text { Multidisciplinary } \\ \square & \\ \square\end{array}$




\section{NPF Topic Clusters for Co-authored Publications}

\section{SciVal Topic Clusters in LANL/UCLA collaborations 2009-2018}

Magnetic Fields, Ionospheres,Sunspots

Decay, Quarks, Neutrinos

Inertial Confinement Fusion, Laser Produced Plasmas,Plasmas (Physics)

Galaxies,Stars,Planets

Beams (Radiation), Accelerators,Free Electron Lasers

Detectors,High Energy Physics, Readout Systems

Neutrons,Neutron Sources,Neutron Scattering

Gravitation,Black Holes (Astronomy),Models

Plasmas (Physics), Dust,Acoustics

Radiolysis, Radiation Chemistry,Liquefied Gases

Detonation,Shock Waves, Explosives

Synthetic Aperture Radar,Radar,Radar Imaging

Student, Ethics, Integrity |

SQUIDs,Superconducting Devices,Electric Potential |

Genes, Gene Regulatory Networks, Gene Expression |

Finite Element Method,Galerkin Methods, Errors |

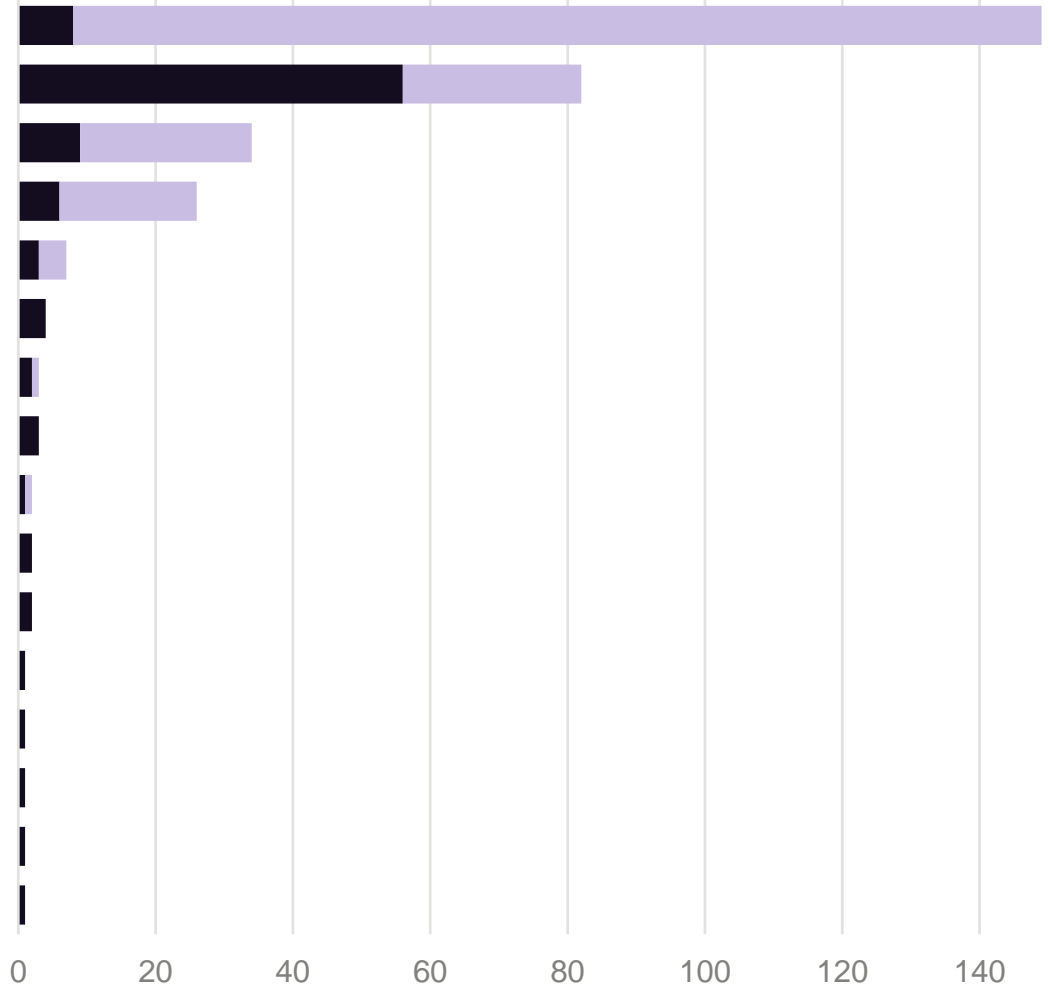

- With NPF Author without NPF author 


\section{LDRD Projects (UCLA Collaborators-NPF Technical Categories)}

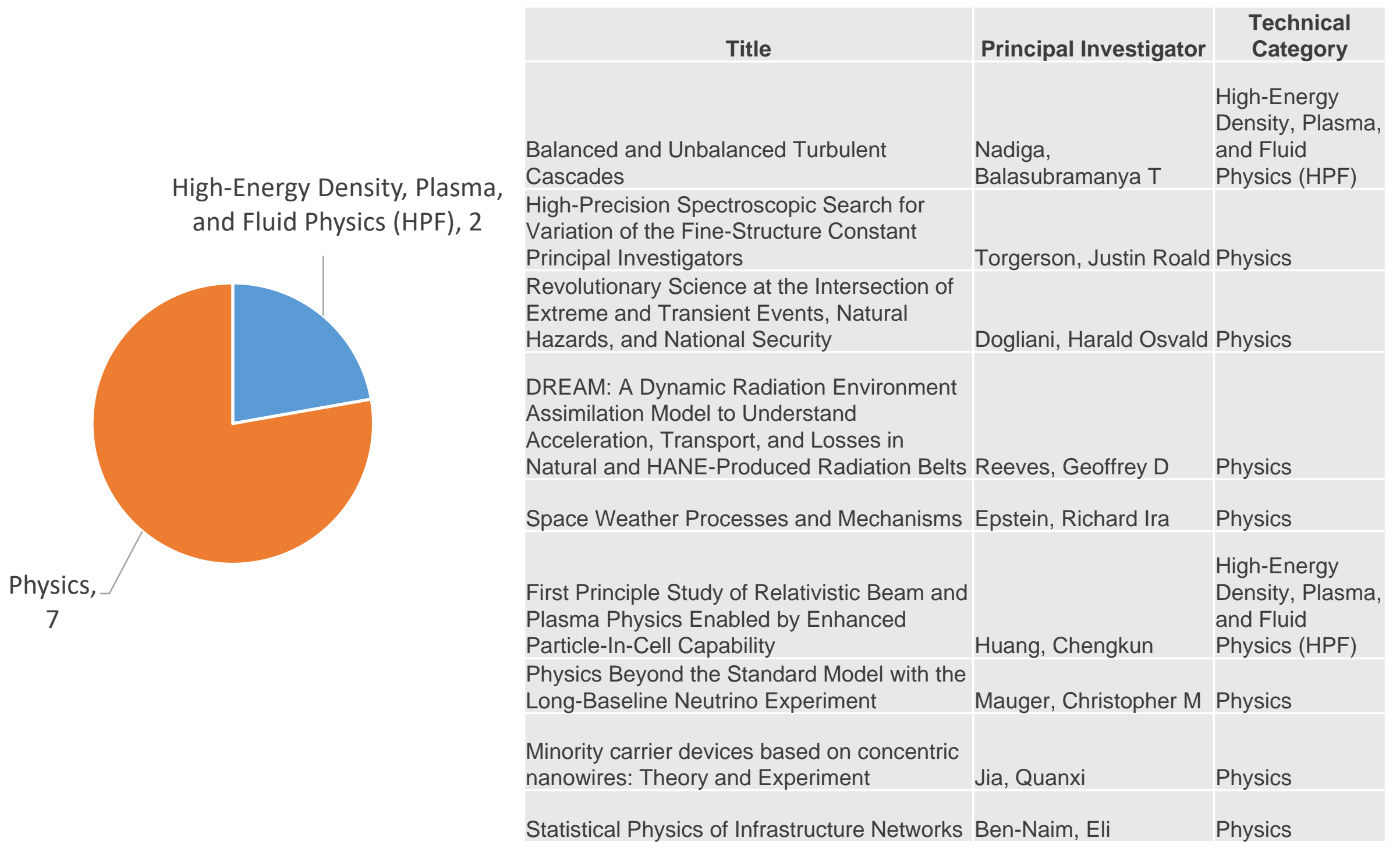




\section{Co-authors by Institution (Top 10)}

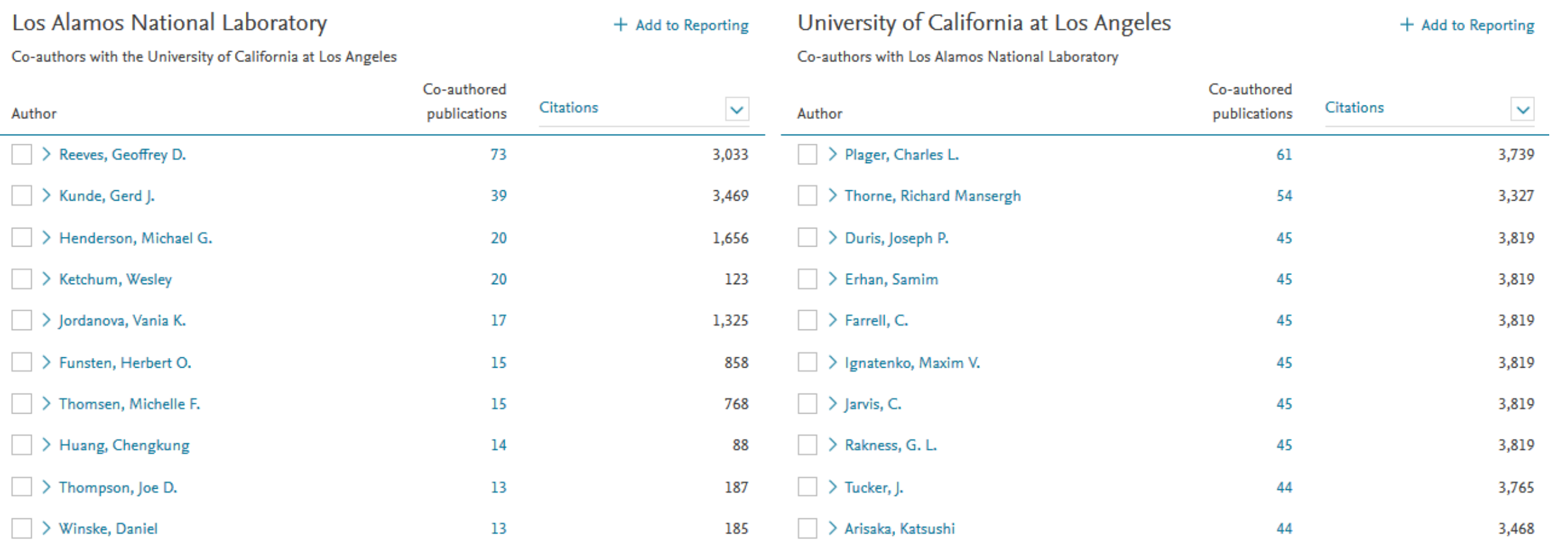




\section{Patent Citations in Co-authored Publications}

Citing-Patents Count

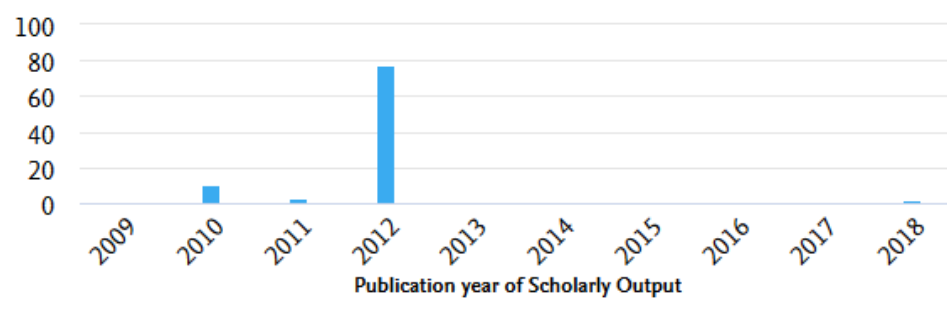

\section{Patent-Cited Scholarly Output}

6
5
4
3
2
1
0

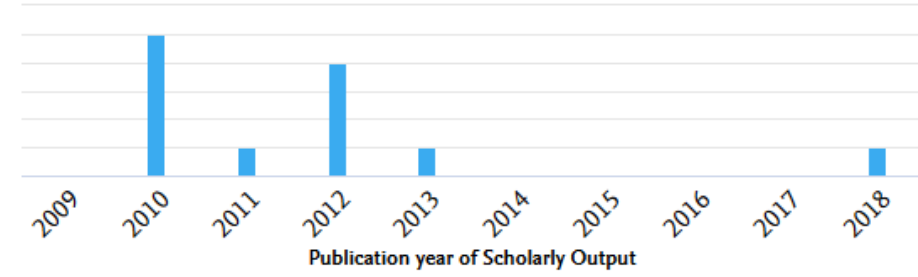

\section{3}

count of patents citing the Scholarly Output published in Publications co-authored by Los Alamos National Laboratory and the University of California at Los Angeles | 2009 to 2018

何 View list of patents

$\underline{\text { Learn more about this metric } \lambda}$

12

count of Scholarly Outputs in Publications co-authored by Los Alamos National Laboratory and the University of California at Los Angeles | 2009 to 2018 that have been cited in patents

View list of publications

Learn more about this metric $\lambda$ 